\title{
REFERENCES
}

1. E. P. Lane, $A$ treatise on projective differential geometry, Chicago, 1942.

2. E. Bompiani, Alcune proprietd proiettivo-differenziali dei sistemi di rette negli iperspazi, Rend. Circ. Mat. Palermo vol. 37 (1914) pp. 305-331.

The University of Chicago

\section{THE RECIPROCAL OF A CONTINUED FRACTION}

\section{W. T. SCOTT}

Stieltjes [3, Chapter X], ${ }^{1}$ and later Rogers [2], gave formulas by means of which the reciprocal continued fractions for continued fractions of a certain class may be determined. We give below a theorem which extends the class of continued fractions to which this reciprocal transformation is applicable ${ }^{2}$ moreover, the theorem is stated in terms of certain parameters which facilitate the transformation.

From results of [1] it is known that there is a unique, one-to-one correspondence between formal power series, $F(z)=1+\sum_{1}^{\infty} c_{p} / z^{p}$, and $C$-fractions,

$$
F(z) \sim 1+\frac{a_{1}}{z^{\beta_{1}}}+\frac{a_{2}}{z^{\beta_{2}}}+\cdots+\frac{a_{n}}{z^{\beta_{n}}}+\cdots,
$$

where the $\beta_{p}$ are integers, $\beta_{0}=0, \beta_{p-1}+\beta_{p}>0$, and $a_{p} \neq 0, p=1,2, \cdots$, $n$, or $p=1,2, \cdots$, according as the $C$-fraction does or does not terminate. The power series for the $p$ th approximant of the $C$-fraction agrees with $F(z)$ for the first $\left(\sigma_{p-1}+\sigma_{p}\right)$ terms, where $\sigma_{p}=\beta_{0}$ $+\beta_{1}+\cdots+\beta_{p}$; this property characterizes the correspondence (1).

For simplicity we put $a_{2 n}=0$ in case a $C$-fraction terminates with the $(2 n-1)$ th partial quotient. Our result is stated below for a class of terminating $C$-fractions. In the nonterminating case the conditions of the theorem must. hold for every index, $n$; consequently, a statement of our result in this case is obtained by replacing $n$ by $\infty$.

Presented to the Society, November 25, 1949; received by the editors February 1, 1952.

${ }^{1}$ Numbers in brackets refer to the bibliography at the end of the paper.

2 Another theorem on reciprocals of $C$-fractions was given by Evelyn Frank, Amer. J. Math. vol. 68 (1946) pp. 89-108. The domain of application of the two theorems does not overlap. 
ThEOREM. The correspondence

$$
F(z) \sim 1+\frac{a_{1}}{z^{\beta_{1}}}+\frac{a_{2}}{1}+\cdots+\frac{a_{2 n-1}}{z^{\beta_{2 n-1}}}+\frac{a_{2 n}}{1}
$$

implies the correspondence

$$
\frac{1}{F(z)} \sim 1+\frac{a_{1}^{*}}{z^{\beta_{1}}}+\frac{a_{2}^{*}}{1}+\cdots+\frac{a_{2 n-1}^{*}}{z^{\beta_{2 n-1}}}+\frac{a_{2 n}^{*}}{1}
$$

if and only if the correspondence (2) has the form

$$
\begin{aligned}
F(z) \sim 1 & +\frac{2 g_{1}}{z^{\beta_{1}}}+\frac{g_{1}\left(g_{2}-1\right)}{1}+\cdots+\frac{\left(g_{2 n-2}+1\right) g_{2 n-1}}{z^{\beta_{2 n-1}}} \\
+ & \frac{g_{2 n-1}\left(g_{2 n}-1\right)}{1},
\end{aligned}
$$

where $g_{2 p-1} \neq 0, p=1,2, \cdots, n$, and $g_{2 p}^{2} \neq 1, p=1,2, \cdots, n-1$. In this case (3) has the form

$$
\begin{aligned}
\frac{1}{F(z)} \sim 1 & -\frac{2 g_{1}}{z^{\beta_{1}}}+\frac{g_{1}\left(g_{2}+1\right)}{1}+\cdots+\frac{\left(g_{2 n-2}-1\right) g_{2 n-1}}{z^{\beta_{2 n-1}}} \\
+ & \frac{g_{2 n-1}\left(g_{2 n}+1\right)}{1},
\end{aligned}
$$

which may be obtained from (4) by replacing $g_{p}$ by $-g_{p}$.

We begin the proof by noting that the $p$ th numerator, $A_{p}(z)$, and denominator, $B_{p}(z)$, of (2), which are the solutions of

$$
y_{p}=z^{\beta_{p}} y_{p-1}+a_{p} y_{p-2}, \quad p=1,2, \cdots, 2 n, \beta_{2 p}=0,
$$

for which $y_{-1}=1, y_{0}=1$, and $y_{-1}=0, y_{0}=1$, respectively, are polynomials in $z$ of degree $\sigma_{p}$ with leading coefficient unity. Since

$$
A_{p}(z) B_{p-1}(z)-A_{p-1}(z) B_{p}(z)=(-1) a_{1} a_{2} \cdots a_{p}, p=1,2, \cdots, 2 n,
$$

it follows that the $p$ th approximant of $(2), A_{p}(z) / B_{p}(z)$, is irreducible. Also $B_{2 p-1}(0)=0, A_{2 p-1}(0) \neq 0, p=1,2, \cdots, n$, while $B_{2 p}(0) \neq 0$. $p=1,2, \cdots, n-1$, and $B_{2 n}(0)=0$ if and only if $a_{2 n}=0$. Similar remarks apply for the numerators, $A_{p}^{*}(z)$, and denominators, $B_{p}^{*}(z)$, of (3).

If (2) implies (3), then

$$
F(z) \sim \frac{1}{1}+\frac{a_{1}^{*}}{z^{\beta_{1}}}+\frac{a_{2}^{*}}{1}+\cdots+\frac{a_{2 n-1}^{*}}{z^{\beta_{2 n-1}}}+\frac{a_{2 n}^{*}}{1},
$$


and the odd contraction of the continued fraction on the right gives (7) $1-\frac{a_{1}^{*}}{z^{\beta_{1}}+a_{1}^{*}+a_{2}^{*}}-\frac{a_{2}^{*} a_{3}^{*}}{z^{\beta_{3}}+a_{3}^{*}+a_{4}^{*}}-\cdots-\frac{a_{2 n-2}^{*} a_{2 n-1}^{*}}{-z^{\beta_{2 n-1}}+a_{2 n-1}+a_{2 n}}$, whose $p$ th approximant is $B_{2 p}^{*}(z) / A_{2 p}^{*}(z)$. The even contraction of the $C$-fraction of (2) is

$$
1+\frac{a_{1}}{z^{\beta_{1}}+a_{2}}-\frac{a_{2} a_{8}}{z^{\beta_{3}}+a_{3}+a_{4}}-\cdots-\frac{a_{2 n-2} a_{2 n-1}}{z^{\beta_{2 n-1}}+a_{2 n-1}^{*}+a_{2 n}^{*}}
$$

whose $p$ th approximant is $A_{2 p}(z) / B_{2 p}(z)$, and from earlier remarks it follows that

$$
A_{2 p}^{*}(z)=B_{2 p}(z), \quad B_{2 p}^{*}(z)=A_{2 p}(z), \quad p=1,2, \cdots, n .
$$

Since the continued fractions (7) and (8) are identical, we deduce the necessity of the conditions

$$
\begin{aligned}
& a_{1}^{*}=-a_{1}, \quad a_{1}^{*}+a_{2}^{*}=a_{2}, \\
& a_{2 p}^{*} a_{2 p+1}^{*}=a_{2 p} a_{2 p+1}, \quad a_{2 p+1}^{*}+a_{2 p+2}^{*}=a_{2 p+1}+a_{2 p+2}, \\
& p=1,2, \cdots, n-1 \text {, }
\end{aligned}
$$

which are precisely the formulas given by Rogers for the case $\beta_{2 p-1}=1, p=1,2, \cdots, n$, and are equivalent to the formulas of Stieltjes for this case.

Continuing with the proof, we note that $A_{2 p-1}(0) \neq 0, p=1,2, \cdots$, $n, B_{2 p}^{*}(0) \neq 0, p=0,1, \cdots, n-1$; hence by $(9), A_{p}(0) \neq 0, p$ $=0,1, \cdots, 2 n-1$, when (2) implies (3). ${ }^{3}$ Then by (6)

$$
\begin{aligned}
& a_{2 p-1}=A_{2 p-1}(0) / A_{2 p-8}(0)=\left(g_{2 p-2}+1\right) g_{2 p-1}, \\
& p=2,3, \cdots, n, a_{1}=2 g_{1}, \\
& a_{2 p}=\left[A_{2 p}(0)-A_{2 p-1}(0)\right] / A_{2 p-2}(0)=g_{2 p-1}\left(g_{2 p}-1\right), \\
& p=1,2, \cdots, n,
\end{aligned}
$$

where

$$
\begin{array}{r}
g_{2 p-1}=A_{2 p-1}(0) / 2 A_{2 p-2}(0), \quad g_{2 p}=-1+2 A_{2 p}(0) / A_{2 p-1}(0), \\
p=1,2, \cdots, n ;
\end{array}
$$

thus the correspondence (2) may be written in the form (4) where

${ }^{3}$ When $\beta_{2 p-1}=1$ and the continued fractions are nonterminating, a theorem of H. S. Wall, Trans. Amer. Math. Soc. vol. 48 (1940) p. 168, is equivalent to the statement that (2) implies (3) if and only if $A_{p}(0) \neq 0, p=1,2, \cdots$. 
$g_{2 p-1} \neq 0, p=1,2, \cdots, n$, and $g_{2 n}^{2} \neq 1, p=1,2, \cdots, n-1$. It is now easy to show by induction that for these $a_{p}, p=1,2, \cdots, 2 n$, the numbers $a_{p}^{*}$ obtained from them by replacing $g_{p}$ by $-g_{p}$, $p=1,2, \cdots, 2 n$, are solutions of $(10)$ for which $a_{p}^{*} \neq 0, p=1,2, \cdots$, $2 n-1$; consequently the correspondence (3) may be written in the form (5).

Conversely, if (4) holds, the partial numerators $a_{p}$ and $a_{p}^{*}$ of the continued fractions in (4) and (5) satisfy (10). Then the continued fractions (7) and (8) are identical and (9) holds, from which it is readily seen that the left member of the correspondence (5) is $1 / F(z)$. Thus a correspondence (2) which can be written in the form (4) implies a correspondence of the form (3), and the proof of the theorem is complete.

It is readily found that the present theorem is applicable to a large class of continued fractions obtained from the continued fractions of Gauss and of Heine. For example the expansion of $F(a,-b, a ;-x) / F(a, 1-b, 1+a ;-x)$ is (cf. [4, p. 337])

$$
\begin{aligned}
1+\frac{\frac{a(a+b)}{a(a+1)}}{1}+ & +\frac{\frac{1 \cdot(1-b)}{(a+1)(a+2)} x}{1}+\frac{\frac{(a+1)(a+b+1)}{(a+2)(a+3)} x}{1} \\
& +\frac{\frac{2(2-b)}{(a+3)(a+4)} x}{1}+\cdots,
\end{aligned}
$$

which converges in the $x$-plane cut from -1 to $-\infty$, except perhaps for isolated points (cf. [4, p. 339]). The parameters of the theorem are found to be $g_{2 p+1}=(a+b) / 2(a+2 n-1), g_{2 p}=1+2 p(p-b) /(a+b)$ $\cdot(a+2 p), p=1,2, \cdots$, and application of the theorem with $z$ replaced by $1 / x$ yields the new expansion,

$$
\begin{gathered}
1-\frac{\frac{a+b}{a+1} x}{1}+\frac{\frac{(a+1)(a+b+1)}{(a+1)(a+2)} x}{1}+\frac{\frac{1 \cdot(1-b)}{(a+2)(a+3)} x}{1} \\
+\frac{\frac{(a+2)(a+b+2)}{(a+3)(a+4)} \times \frac{2(2-b)}{(a+4)(a+5)} x}{1}+\frac{\frac{(a+1}{1}+\cdots}{}
\end{gathered}
$$

for the reciprocal of the original series. It is easily shown that the new expansion converges in the $x$-plane cut from -1 to $-\infty$, except perhaps at isolated points. 


\section{BIBLIOGRAPHY}

1. Walter Leighton and W. T. Scott, A general continued fraction expansion, Bull. Amer. Math. Soc. vol. 45 (1939) pp. 596-605.

2. L. J. Rogers, On the representation of certain asymptotic series as convergent continued fractions, Proc. London Math. Soc. (2) vol. 4 (1907) pp. 72-89.

3. T. J. Stieltjes, Recherches sur les fractions continues, Oeuvres, Groningen, 1914-1918, vol. 2, pp. 402-566.

4. H. S. Wall, Analytic theory of continued fractions, New York, 1948.

\section{NORTHWESTERN UNIVERSITY}

